\title{
COLESTEROL, 7-CETOCOLESTEROL E 25-HIDROXICOLESTEROL EM MAIONESE
}

\author{
Isabel Cristina MORALES-AIZPURÜA, Alfredo TENUTA-FILHO
}

\section{RESUMO}

A maionese é um alimento com predominância lipídica característica. O sistema antioxidante artificial usado resulta em longa vida-de-prateleira do produto. Mas não existem conhecimentos suficientes quanto à estabilidade oxidativa do colesterol incorporado à maionese, através do ovo. Neste trabalho foi confirmada a oxidação do colesterol contido na maionese, pela presença do 7 -

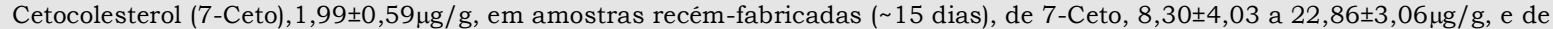
25-Hidroxicolesterol, 2,21 $\pm 1,94$ a 13,98 $2,72 \mu \mathrm{g} / \mathrm{g}$, em amostras comerciais (A,B,C,D e E) com tempos de fabricação de 30 a 210 dias, e de 7-Ceto, $8,44 \pm 6,78 \mathrm{\mu g} / \mathrm{g}$, em amostras coletadas em domicílios (produto em uso, mantido em geladeira), quantificados por HPLC. O somatório dos óxidos de colesterol (OsC) analisados nas amostras A/E correspondeu a 2,07-4,94\% em relação ao colesterol $(50,66 \pm 3,08-73,36 \pm 3,53 \mathrm{mg} / 100 \mathrm{~g})$. Os resultados obtidos são significativos em relação às atividades biológicas deletérias atribuídas aos $\mathrm{OsC}$, e quanto ao aspecto tecnológico envolvido na produção da maionese.

Palavras-chave: colesterol; óxidos de colesterol; 7-cetocolesterol; 25-hidroxicolesterol; maionese.

\section{SUMMARY}

CHOLESREROL, 7-KETOCHOLESTEROL AND 25-HYDROXYCHOLESTEROL IN MAYONNAISE. The antioxidant system used in mayonnaise production is efficient mainly in relation to oxidation of unsaturated fatty acids. Consequently the shelf-life of the product is large (six months). However very little is known about the stability of cholesterol present in mayonnaise introduced with the eggs (raw material). The oxidation of cholesterol in mayonnaise was confirmed quantifying 7-Ketocholesterol (7-Keto) and 25Hydroycholesterol $(25-\mathrm{OH})$ using HPLC. The following occurrences were observed: 7 -Keto, $1.99 \pm 0.59 \mu \mathrm{g} / \mathrm{g}$, in recently manufactured mayonnaise (approximately 15 days), 7 -Keto, $8.30 \pm 4.03$ to $22.86 \pm 3.06 \mu \mathrm{g} / \mathrm{g}$, and $25-\mathrm{OH}, 2.21 \pm 1.94$ to $13.98 \pm 2.72 \mu \mathrm{g} / \mathrm{g}$, in mayonnaise samples (A,B,C,D and E) with 30 to 210 days after production, and 7-Keto, $8.44 \pm 6.78 \mu \mathrm{g} / \mathrm{g}$, in mayonnaise samples collected at domiciles (products in use stored under refrigeration). The sum between 7-Keto and 25-OH from A/E samples amounted to $2.07-4.94 \%$ in relation to cholesterol $(50.66 \pm 3.08-73.36 \pm 3.53 \mathrm{mg} / 100 \mathrm{~g})$. Results here obtained are relevant in relation to deleterious biological activity attributed to cholesterol oxides and also by the technological aspects involved in mayonnaise manufacture.

Keywords: cholesterol; cholesterol oxides; 7-ketocholesterol; 25-hidroxycholesterol; mayonnaise.

\section{1 - INTRODUÇÃO}

O colesterol é um lipídio insaturado e suscetivel à oxidação, influenciada pelo oxigênio e outros fatores como a atividade de água, luz, temperatura elevada, radiação ionizante, radicais livres e íons metálicos. O perfil dos óxidos de colesterol (OsC) formados e as quantidades correspondentes dependem das características dos alimentos e interações entre seus componentes e produtos de decomposição, durante o processamento (inclusive em nível doméstico) e/ou estocagem [24].

Os OsC presentes em alimentos são compostos de interesse tanto tecnológico como clínico, pela relação com processos citotóxicos, angiotóxicos, aterogênicos, mutagênicos e carcinogênicos, além de outros efeitos deletérios. Dezenas de produtos da oxidação do colesterol têm sido identificadas, sendo os mais freqüentemente encontrados em alimentos o 7-Cetocolesterol (7-Ceto), 20-

Recebido para publicação em 18/05/2004. Aceito para publicação em 25/07/2005(001349).

Departamento de Alimentos e Nutrição Experimental, Faculdade de Ciências Farmacêuticas, Universidade de São Paulo. Endereço: Av. Lineu Prestes, 580, bloco 14. CEP: 05508-900, São Paulo, Brasil. Email:eetenuta@usp.br

A quem a correspondência deve ser enviada.
Hidroxicolesterol (20-OH), 25-Hidroxicolesterol (25-OH), $7 \alpha$-Hidroxicolesterol $(7 \alpha-\mathrm{OH}), 7 \beta$-Hidroxicolesterol $(7 \beta-$ $\mathrm{OH})$, Colesterol-5,6 6 -epóxido (5,6 6 -epóxido), Colesterol-

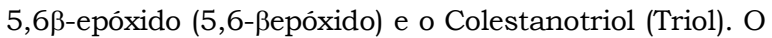
7-Ceto ocorre em concentrações relativamente altas e, em função disso, tem sido proposto usá-lo como indicador da oxidação do colesterol. Ao contrário, o 25-OH e o Triol são os de menor ocorrência. Os OsC são absorvidos através dos alimentos e entram na circulação sangüínea como contaminantes $[1,3,4,5,13,14,16,17,18,20$, 26, 30, 32, 34, 35].

A maionese é um alimento com alto teor lipídico e um produto comercial com longa vida-de-prateleira (seis meses). É protegida por um sistema antioxidante artificial voltado à estabilidade dos ácidos graxos insaturados, mas que não se destinaria a prevenir especificamente a ocorrência da oxidação do colesterol, veiculado pelo ovo usado como matéria-prima do produto. Ao que parece, somente SARANTINOS, O'DEA \& SINCLAIR [29] examinaram a maionese quanto à incidência de OsC. O estudo foi feito com um número muito reduzido de amostras $(\mathrm{n}=3)$, tendo sido quantificados o $7 \alpha-\mathrm{OH}, 20-\mathrm{OH}, 7-\mathrm{Ceto}$, Triol e 25-OH. Os referidos autores relataram a ocorrência do $4 \beta$-Hidroxicolesterol, um óxido de menor freqüência, em quantidades apreciáveis comparadas com as dos demais. De acordo com a literatura, muito pouco ou quase nada é sabido quanto à estabilidade oxidativa do colesterol contido na maionese. 
No presente trabalho, amostras comerciais de maionese e do produto em uso domiciliar foram analisadas em relação ao colesterol e à ocorrência do 7-Ceto, como indicador da oxidação do referido esterol, e do 25-OH, um dos OsC mais aterogênicos encontrados em alimentos.

\section{2 - MATERIAL E MÉTODOS}

\section{1 - Material}

\subsection{1 - Amostras}

Três grupos de amostras de maionese foram analisadas (em triplicata): 1) cinco amostras distintas $(n=5)$ da mesma marca comercial, codificada como A, com 15 (13-17) dias de fabricação, obtidas diretamente do fabricante; 2) três amostras ( $n=3)$ aleatórias, com tempos de fabricação variados, de cada uma das cinco principais marcas oferecidas comercialmente, codificadas como A, B, C, D e E; e, 3) dezamostras (n=10) aleatórias que estavam em uso domiciliar, mantidas em geladeira. Exceto uma, as demais amostras analisadas estavam dentro do prazo de validade, de 180 dias. Os tempos de fabricação das amostras A/E eram respectivamente: A, de 30, 45 e 90 dias; $\mathrm{B}$, de 30, 90 e 120 dias; $\mathrm{C}$, de 30, 90 e 90 dias; D, de 30,120 e 180 dias; e, E, de 120, 180 e 210 dias.

\subsection{2 - Reagentes}

O hexano (Aldrich) e isopropanol (Sigma) usados tinham grau analítico HPLC e os demais reagentes grau ACS. O colesterol, 6-cetocolestanol (padrão interno), 7Ceto e 25-OH foram obtidos da Sigma Chemical Co.

\section{2 - Extração e saponificação dos lipidios totais da maionese dos lipidios totais}

As amostras de maionese $(0,25$ a $1,0 \mathrm{~g})$ foram reconstituídas a um teor de umidade de $80 \mathrm{~g} / 100 \mathrm{~g}$, por adição de água destilada e homogeneizadas, transferidas para um funil de separação, com $50 \mathrm{~mL}$ de água destilada, e extraídas com $20 \mathrm{~mL}$ de clorofórmio/metanol (v/v, 2:1), por três vezes consecutivas [10]. A saponificação, que se deu após a separação e evaporação do clorofórmio a $40^{\circ} \mathrm{C}$, sob vácuo, foi realizada com adição ao resíduo de $20 \mathrm{~mL}$ de $\mathrm{KOH} 1 \mathrm{M}$ em metanol, mantendo a mistura por 18 horas, a $25-30^{\circ} \mathrm{C}$, com agitação mecânica nas duas primeiras e nas duas últimas horas do procedimento. À mistura obtida foram incorporados $10 \mathrm{~mL}$ de água destilada e procedida a extração da fração insaponificável em funil de separação, por três vezes consecutivas, com $10 \mathrm{~mL}$ de hexano. A fração orgânica foi lavada com $10 \mathrm{~mL}$ de $\mathrm{KOH} 1 \mathrm{M}$ em metanol e, por três vezes consecutivas, com $10 \mathrm{~mL}$ de água destilada. $\mathrm{O}$ extrato obtido foi filtrado em papel Whatman 1 contendo sulfato de sódio anidro e o material retido no filtro lavado com $20 \mathrm{~mL}$ de hexano [8].

\section{3 - Quantificação do colesterol e óxidos de co- lesterol}

O extrato de hexano anteriormente obtido foi evapo- rado em evaporador rotatório, a $40^{\circ} \mathrm{C}$, sob vácuo. O resíduo foi suspenso em 1 a $2 \mathrm{~mL}$ da fase móvel, filtrado em membrana durapore com $0,45 \mu \mathrm{m}$ de diâmetro (MillexMillipore) e injetado (20-50 $\mu \mathrm{L})$ automaticamente no sistema de HPLC (Shimadzu, LC-10ADVP). Foi usada coluna de sílica, $\mu$-Porasil, de $30 \times 0,39 \mathrm{~cm}$, com poro de $10 \mu \mathrm{m}$ de diâmetro (WATERS Associates), em fase normal. As fases móveis utilizadas foram misturas de hexano/isopropanol (v/v),: 94:6 para o colesterol, 97:3 para o $25-\mathrm{OH}$ e $93: 7$ para o 7 -Ceto, com fluxo de $1 \mathrm{~mL} / \mathrm{min}$. O colesterol e o $25-\mathrm{OH}$ foram monitorados a $206 \mathrm{~nm}$ e o 7 -Ceto a $233 \mathrm{~nm}$, com detector de fotodiodos (UV-Visivel). A identificação dos picos de colesterol e dos OsC foi realizada por comparação dos tempos de retenção em relação aos padrões correspondentes. Na quantificação do colesterol e 25-OH foi usada padronização externa e, no caso do 7Ceto, interna e externa, com o 6-cetocolestanol como padrão interno [10, 22].

A validação do método foi estabelecida por diferentes parâmetros [19, 25, 27]. A recuperação dos analitos (adicionados à amostra antes da extração lipídica) foi de 90$96 \%(n=6) ;$ a repetitividade (medida pelo coeficiente de variação) de 7,25 a 9,29 \% (n=8); e, a linearidade da curva

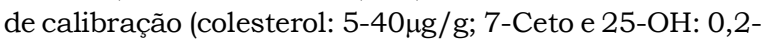
$1,2 \mu \mathrm{g} / \mathrm{g}$ ) (representada pelo coeficiente de correlação $\mathrm{r}^{2}$ ), de 0,993-0,999. Os limites de detecção e de quantificação foram de $6,67 \times 10^{\circ}$ e $2,22 \times 10^{\circ} \mathrm{g}$ para o $25-\mathrm{OH}$, $2,02 \times 10^{\circ}$ e $6,73 \times 10^{\circ}$ g para o 7 -Ceto e $1,09 \times 10^{\circ}$ e $3,62 \times 10^{-1}$ para o colesterol.

A estrutura dos OsC de colesterol foi confirmada, de acordo com SEVANIAN et al. [31], por cromatografia a gás-espectrometria de massa.

\section{4 - Umidade, lipidios totais, valor de peróxido e valor de $\mathbf{p H}$}

A umidade e o valor de peróxido (VP) foram determinados segundo a AMERICAN OIL CHEMISTS' SOCIETY [2], os lipídios totais de acordo com BRASIL [7] e o valor de $\mathrm{pH}$ medido diretamente na amostra homogeneizada, usando potenciômetro da Procyon, modelo SA 720.

\section{5 - Análise estatística}

Os dados obtidos foram analisados pela análise de variância, ANOVA (Microsoft Excel) $(p=0,05)$.

\section{3 - RESULTADOS E DISCUSSÃO}

\section{1 - Umidade, lipidios totais e valores de peró- xido e de pH, em maionese comercial}

Na Tabela 1 podem ser observados os resultados obtidos para umidade, lipídios totais e valores de peróxido e de $\mathrm{pH}$.

A umidade observada, de 15,87-20,57g/100g, está de acordo com o que foi relatado por CHIRIFE et al. [9] para maionese, de 15,8-18,4g/100g. Os lipídios totais encontrados, de 71,27-77,28g/100g, são concordantes 
TABELA 1 - Umidade, lipídios totais e valores de peróxido e de $\mathrm{pH}$ em amostras de maionese comercial

\begin{tabular}{ccccc}
\hline Amostras & $\begin{array}{c}\text { Umidade } \\
(\mathrm{g} / 100 \mathrm{~g})\end{array}$ & $\begin{array}{c}\text { Lipídios } \\
\text { totais } \\
(\mathrm{g} / 100 \mathrm{~g})\end{array}$ & $\begin{array}{c}\text { Valor de } \\
\text { peróxido } \\
(\mathrm{mEq} \mathrm{O} / \mathrm{kg})\end{array}$ & $\begin{array}{c}\text { Valor de } \\
\mathrm{pH}\end{array}$ \\
\hline RF & $16,97 \pm 0,14$ & $75,75 \pm 0,77$ & $0,75 \pm 0,01$ & $3,68 \pm 0,01$ \\
$\mathrm{~A}$ & $16,55 \pm 0,10^{\mathrm{a}}$ & $77,26 \pm 0,26^{\mathrm{a}}$ & $1,93 \pm 0,09^{\mathrm{a}}$ & $3,75 \pm 0,01^{\mathrm{a}}$ \\
$\mathrm{B}$ & $20,57 \pm 0,90^{\mathrm{b}}$ & $71,27 \pm 1,78^{\mathrm{b}}$ & $3,83 \pm 0,72^{\mathrm{b}}$ & $3,52 \pm 0,02^{\mathrm{b}}$ \\
$\mathrm{C}$ & $17,10 \pm 2,33^{\text {abc }}$ & $75,02 \pm 0,94^{\mathrm{c}}$ & $3,07 \pm 1,89^{\mathrm{a}} \mathrm{b}$ & $3,70 \pm 0,08^{\mathrm{c}}$ \\
D & $17,14 \pm 1,37^{\mathrm{a}}$ & $73,69 \pm 0,80_{\mathrm{cb}}$ & $3,87 \pm 0,71^{\mathrm{b}}$ & $3,77 \pm 0,03^{\mathrm{a}} \mathrm{d}$ \\
E & $15,87 \pm 0,28^{\mathrm{c}}$ & $77,28 \pm 2,25^{\mathrm{c}}$ & $4,34 \pm 0,11^{\mathrm{b}}$ & $3,80 \pm 0,01^{\triangleleft}$ \\
\hline
\end{tabular}

Médiatdesvio-padráo. RF: Recém-fabricadas ( 15 dias) ( $\mathrm{n}=5)$. Tempos de fabricação das amostras A/E (n-3): A ( 30,45 e 90 dias), $B$ ( 30,90 e 120 dias), C ( 30,90 e 90 dias), $\mathrm{D}(30,120 \mathrm{e} 180$ dias), $\mathrm{E}(120,180 \mathrm{e} 210$ dias). Letras sobrescritas iguais indicam que as diferenças observadas nas colunas náo séo estatisticamente significativas, ao contrário
das letras sobrescritas diferentes.

com a legislação brasileira, que estabelece um minimo de $65 \mathrm{~g}$ de óleo vegetal comestivel/100g do produto [6], ao mesmo tempo que se assemelharam com o que foi mencionado por CHIRIFE et al. [9], de 77,4-79,1g/100g, e STAUFFER [33], de 77-82 g/100g, porém superiores em relação ao resultado de SARANTINOS, O'DEA \& SINCLAIR [29], 67,9g/100g.

O valor médio de peróxido nas amostras com $\sim 15$ dias de fabricação (RF) foi bem menor que os das demais (0,75 e entre 1,93 e 4,34 $\mathrm{mEq}$ de $\mathrm{O}_{2} / \mathrm{kg}$, respectivamente), sendo que, os níveis de peróxido detectados estavam abaixo do limite previsto em legislação, de $10 \mathrm{mEq}$ de $\mathrm{O}_{2} / \mathrm{kg}$ de óleo (refinado) [28].

Os valores de $\mathrm{pH}$ foram de 3,52-3,80, abaixo do máximo aceitável ( $\mathrm{pH} 4,2)$ para que a maionese mantenha a estabilidade microbiológica, segundo a prática industrial [15]. O pH baixo pode favorecer a oxidação de ácidos graxos, pela liberação do ferro existente no ovo na interface óleo/água. Em razão disso, o metal é complexado por aditivos específicos adicionados ao produto [15].

\section{2 - Colesterol, 7-cetocolesterol e 25- hidroxicolesterol em amostras de maionese co- mercial e em uso domiciliar}

$\mathrm{Na}$ Tabela 2 podem ser vistos os valores para o colesterol, 7-Ceto e 25-OH, determinados em maionese comercial e em uso domiciliar.

Quantidades apreciáveis de colesterol, de 50,66 a $73,36 \mathrm{mg} / 100 \mathrm{~g}$, foram encontradas nas amostras de maionese analisadas. Estes resultados são muito semelhantes aos de FEELEY, CRINER \& WATT [11], de 50 a $70,0 \mathrm{mg} / 100 \mathrm{~g}$, mas muito superiores ao de SARANTINOS, O'DEA \& SINCLAIR [29], de $18,3 \mathrm{mg} / 100 \mathrm{~g}$, cujo valor sugere uma menor proporção proposital de ovo no produto.

O teor médio de 7 -Ceto, de 1,99 $\pm 0,59 \mu \mathrm{g} / \mathrm{g}$, nas amostras com $~ 15$ dias de fabricação (RF) foi significativamente menor que os das demais (A/E), de 8,30-22,86 $\mathrm{g} / \mathrm{g}$, cujo tempo de fabricação entre estas variou de 30 a 210 dias. O 25-OH não foi encontrado nas amostras RF. A inci-
TABELA 2 - Colesterol, 7-Cetocolesterol (7-Ceto) e 25Hidroxicolesterol (25-OH) em amostras de maionese comercial e em uso domiciliar

\begin{tabular}{ccccc}
\hline Amostras & $\begin{array}{c}\text { Colesterol } \\
\mathrm{mg} / 100 \mathrm{~g}(\mathrm{a})\end{array}$ & $\begin{array}{c}7-\mathrm{Ceto} \\
\mu \mathrm{g} / \mathrm{g}(\mathrm{b})\end{array}$ & $\begin{array}{c}25-\mathrm{OH} \\
\mu \mathrm{g} / \mathrm{g}(\mathrm{c})\end{array}$ & $\begin{array}{c}\mathrm{b}+\mathrm{c} \times 100 / \mathrm{a} \\
(\%)\end{array}$ \\
\hline RF & $71,36 \pm 5,49$ & $1,99 \pm 0,59$ & n.d & - \\
A & $50,66 \pm 3,08^{\mathrm{a}}$ & $8,30 \pm 4,03^{\mathrm{a}}$ & $2,21 \pm 1,94^{\mathrm{a}}$ & 2,07 \\
B & $61,01 \pm 18,24^{\mathrm{ab}}$ & $17,96 \pm 2,27^{\mathrm{b}}$ & $3,46 \pm 5,71^{\mathrm{ab}}$ & 3,51 \\
C & $50,79 \pm 5,1^{\mathrm{a}}$ & $8,42 \pm 2,21^{\text {ac }}$ & $13,98 \pm 2,72^{\mathrm{c}}$ & 4,41 \\
D & $52,74 \pm 14,13^{\mathrm{ab}}$ & $10,94 \pm 3,29^{\mathrm{a}}$ & $12,57 \pm 1,19^{\mathrm{bc}}$ & 4,46 \\
E & $73,36 \pm 3,53^{\mathrm{b}}$ & $22,86 \pm 3,06^{\mathrm{b}}$ & $13,38 \pm 3,73^{\mathrm{bc}}$ & 4,94 \\
UD & - & $8,44 \pm 6,78$ & - & -
\end{tabular}

Médiatdesvio-padrão. n.d: Não detectado (abaixo do limite de quantificação). b+c $\times 100 / a:$ Tomatório dos OsC em relaçáo ao colesterol. RF: Recém-fabricadas ( 15 dias) (n=5). $\mathrm{C}(30,90 \mathrm{e} 90$ dias), $\mathrm{D}$ ( $30,120 \mathrm{e} 180$ dias), $\mathrm{E}$ ( 120,180 e 210 dias). UD: Amostras em uso domiciliar $(\mathrm{n}=10)$. Letras sobrescritas iguais indicam que as diferenças observadas nas colunas não são estatisticamente significativas, ao contránio das letras sobrescritas diferentes.

dência de 7-Ceto nas amostras RF poderia ter decorrido de sua já presença no ovo, enquanto matéria-prima, e/ou em função processo de obtenção da maionese, considerando que em ovos frescos não tem sido constatado o referido óxido $[29,36]$.

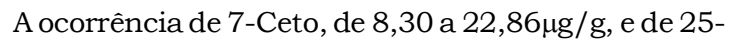

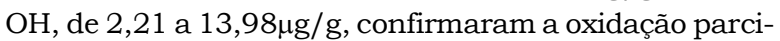
al do colesterol nas amostras $\mathrm{A} / \mathrm{E}$. A diferença observada entre as amostras $\mathrm{RF}$ e as $\mathrm{A} / \mathrm{E}$ poderia estar indicando aumento na concentração dos OsC em função do tempo de fabricação. No entanto, nenhuma correlação significativa pôde ser observada nesse sentido, considerando os óxidos individualmente ou o somatório de ambos. O somatório do 7-Ceto e 25-OH nas amostras A/E correspondeu a 2,07 a 4,94\% em relação ao colesterol. Este resultado é comparável aos dos OsC encontrados em alimentos de expressivo consumo, de $1-2 \%$ até $10 \%$, segundo PANIANGVAIT et al.[26].

A presença de peróxido, de $1,93-4,34 \mathrm{mEqO}_{2} / \mathrm{kg}$ de óleo (Tabela 1), mesmo em níveis aceitáveis ( $<10 \mathrm{mEqO}_{2} / \mathrm{kg}$ de óleo refinado), poderia ter tido influência no processo oxidativo do colesterol contido nas amostras de maionese analisadas. Mas não foi constatada correlação significativa entre os valores de peróxido e as quantidades de 7 -Ceto e $25-\mathrm{OH}$ formadas, somadas entre si ou não (Tabela 2).

O 25-OH é um dos óxidos de menor ocorrência em alimentos, e aquele com maior efeito aterogênico, ao lado do Triol [14]. No entanto, nas amostras A/E seus níveis foram muito elevados comparados aos do 7-Ceto, considerado indicador da oxidação do colesterol, por sua maior presença em alimentos. Os resultados obtidos indicam a participação oxidativa dos carbonos 7 e 25, respectivamente no anel B e na cadeia lateral, da molécula do colesterol. Como apenas dois entre os oito OsC de maior freqüência em alimentos foram quantificados, os resultados da Tabela 2 estariam subestimando a oxidação do colesterol contido na maionese. Por hipótese então poderia ter havido também a ocorrência, mesmo que em menores concentrações, de $7 \alpha-\mathrm{OH}$ e $7 \beta-\mathrm{OH}$, precursores do 7 - 
Ceto, de 20-OH (cadeia lateral), de 5,6 $\alpha$ a-epóxido e 5,6 $\beta$ epóxido (carbonos 5 e 6, no anel B), e de Triol, este último formado por hidratação, a partir dos 5,6-epóxidos ( $\alpha$ e $\beta$ ) $[14,20,21,32]$.

Nas amostras de maionese em uso (UD), das quais 90\% delas corresponderam ao mesmo fabricante da amostra A, os resultados de 7 -Ceto variaram de 1,33 até $22,82 \mu \mathrm{g} / \mathrm{g}$, com um teor médio de $8,44 \pm 6,78 \mu \mathrm{g} / \mathrm{g}$. Consistentes com o fato de serem diferentes entre si, quanto ao tempo de aquisição e uso domiciliar, os resultados não diferiram muito dos encontrados nas amostras de maionese obtidas no comercio local (A/E), que va-

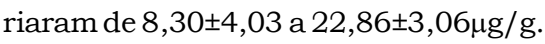

SARANTINOS, O1DEA \& SINCLAIR [29] encontraram $13 \pm 13 \mu \mathrm{g} / \mathrm{g}$ de 7 -Ceto e $9 \pm 4 \mu \mathrm{g} / \mathrm{g}$ de $25-\mathrm{OH}(\mathrm{n}=3)$. Estes resultados e os obtidos no presente trabalho, $8,30 \pm 4,03$ a $22,86 \pm 3,06 \mu g$ de 7 -Ceto/g e 2,21 $\pm 1,94$ a $13,98 \pm 2,72 \mu \mathrm{g}$ de $25-\mathrm{OH} / \mathrm{g}$, são igualmente altos quando comparados aos relatados por GALOBART \& GUARDIOLA [12], para o ovo fresco integral líquido, gema fresca líquida e ovo líquido pasteurizado comercial, que são matérias-primas usadas na produção de maionese. Níveis de até $2,2 \mu \mathrm{g}$ de 7 -Ceto/g foram mencionados para estas matérias-primas, enquanto o 25-OH não foi detectado.

Não sendo conhecidas as quantidades individuais ou total de OsC, que possam representar riscos citotóxi$\cos$, angiotóxicos, aterogênicos, mutagênicos, carcinogênicos, e/ou de outras manifestações possiveis [17], os resultados do presente estudo apontam como significativa a contribuição da maionese com respeito à ingestão de 7Ceto e principalmente de $25-\mathrm{OH}$.

O processo oxidativo do colesterol sugerido no presente trabalho pode ter tido a influência do processamento, condições de armazenamento, oxidação coexistente de ácidos graxos e sistema antioxidante com eficiência pouco específica relativa ao colesterol, entre outros fatores [24, 36]. A oxidação do colesterol pode ocorrer mesmo com a maionese sendo mantida sob refrigeração $\left(4^{\circ} \mathrm{C}\right)$ e na ausência de luz [23].

\section{4 - CONCLUSÕES}

Foi confirmada a oxidação do colesterol contido em maionese comercial, pela ocorrência de 7-Cetocolesterol e 25-Hidroxicolesterol no produto recém-fabricado ( 15 dias) e naquele com tempos de fabricação maiores (3-120 dias), o mesmo se dando em relação àquele em uso domiciliar.

\section{5 - REFERÊNCIAS BIBLIOGRÁFICAS}

[1] ADDIS, P.B.; PARK, P.; GUARDIOLA, F.; CODONY, R. Analysis and health effects of cholesterol oxides. In: McDonald R E, Min, DBJ, editors. Food lipids and health. New York: Marcel Dekker. p. 199-240, 1996.

[2] AMERICAN OIL CHEMISTS' SOCIETY. Official methods and recommended practices, $v 1$. Champaign: AOCS.
490 p., 1987.

[3] APPELQVIST, L.A. Oxidized sterols. Bull. Int. Dairy Fed., Brussels, v. 315, p. 52-58, 1996.

[4] BASCOUl, J.; DOMERGUe, N.; CRASTES DE PAULET, A. Intestinal absorption of cholesterol autoxidation products in dietary fats. J. Amer. Oil. Chem. Soc., Champaign, v. 62, p. 623-628, 1985.

[5] BÖSINGER, S.; LUF, W.; BRANDL, E. Oxysterols: their occurrence and biological effects. Int. Dairy J., Amsterdam, v. 3, p.1-33, 1993.

[6] BRASIL. Diário Oficial da União. Leis, decretos, etc. Portaria 12/78 da CNPA. Brasília, 1978.

[7] BRASIL. Ministério da Agricultura. Secretaria Nacional de Defesa Agropecuária. Laboratório Nacional de Referência Animal. Métodos analíticos oficiais para controle de produtos de origem animal e seus ingredientes: métodos físicos e químicos. Brasília: LANARA, v.2, cap. 11, 1981.

[8] CHEN, B.H.; CHEN, Y.C. Evaluation of analysis of cholesterol oxides by liquid chromatography. J. Chromatogr., A, Amsterdam, v. 661, p. 127-136, 1994.

[9] CHIRIFE, J.; VIGO, M.S.; GOMEZ, R.G.; FAVETTO, G.J. Water activity and chemical composition of mayonnaises. J. Food Sci., Chicago, v. 34, n. 6, p. 1658-1659, 1989.

[10] CSALLANY, A.S.; KINDOM, S.E.; ADDIS, B.; LEE, J. HPLC: method for quantitation of cholesterol and four of its major oxidation products in muscle and liver tissues. Lipids, Champaign, v. 24, n. 7, p. 645-651, 1989.

[11] FEELEY, R.M.; CRINER, P.E.; WATT, B.K. Cholesterol content of foods. J. Am. Diet. Assoc., Chicago, v. 61, p. 131-149, 1972.

[12] GALOBART, J.; GUARDIOLA, F. Formation and content of cholesterol oxidation products in egg and egg products. In: GUARDIOLA, F.; DUTTA, P.C.; CODONY, R.; SAVAGE, G.P.,eds. Cholesterol and phytosterol oxidation products. Analysis, occurrence, and biological effects. Champaign: AOAC Press. p. 124-146, 2002 .

[13] GUARDIOLA, F.; CODONY, R.; ADDIS, P.B.; RAFECAS, M.; BOATELLA, J. Biological effects of oxysterols: current status. Food Chem. Toxicol., Oxford, v. 34, n. 2, p. 193211, 1996.

[14] GUARDiOla, F.; CODONY, R.; RAFECAS, M.; BOATELLA, J. Formación de derivados oxidados del colesterol en alimentos. Grasas Aceites, Sevilla, v. 46, n. 3, p. 202-212, 1995.

[15] JACOBSEN, C.; HARTVIGSEN, K.; THOMSEN, M.; HANSEN, L.F.; LUND, P.; SKIBSTED, L.H.; HOLMER, G.; ADLER-MISSEN, J.; MEYER, A.S. Lipid oxidation in fish oil enriched mayonnaise: Calcium disodium ethylene diamine tetracetate, but not gallic acid, strongly inhibited oxidative deterioration. J. Agric. Food Chem., Columbus, v. 49, p. 1009-1019, 2001.

[16] LERCKER, G.; RODRIGUEZ-ESTRADA, M.T. Cholesterol oxidation mechanisms. In: Guardiola F, Dutta PC, Codony R, Savage GP, eds. Cholesterol and phytosterol oxidation products: Analysis, occurrence, and biological effects. Champaign: AOCS Press. p. 1-25, 2002 . 
[17] LINSEISEN, J.; WOLFRAM, G. Absorption of cholesterol oxidation products from ordinary foodstuff in humans. Ann. Nutr. Metab., Basel, v. 42, p. 221-230, 1998a.

[18] LINSEISEN, J.; WOLFRAM, G. Origin, metabolism, and adverse health effects of cholesterol oxidation products. Fett/Lipid, Weinhein, v. 100, n. 6, p. 211-218, 1998b.

[19] LONG, G.L.; WINEFORDNER, J.D. Limit of detection. A closer look at the IUPAC definition. Anal. Chem., Washington, v. 35, n. 7, p. 712A-724A, 1983.

[20] MAERKER, G. Cholesterol autoxidation-current status. J. Am. Oil Chem. Soc., Champaign, v. 64, n. 3, p. 388392, 1987.

[21] MAERKER, G.; JONES, K.C. Gamma-irradiation of individual cholesterol oxidation products. J. Am. Oil. Chem. Soc., Champaign, v. 69, n. 5, p. 451-455, 1992.

[22] MAERKER, G.; NUNGUESSER, E.H.; ZULAK, I.M. HPLC separation and quantitation of cholesterol oxidation products with flame ionization detection. J. Agric. Food Chem., Columbus, v.36, p. 61-63, 1988.

[23] MORALES-AIZPURÚA, I.C.; TENUTA-FILHO, A. Oxidation of cholesterol in mayonnaise during storage. Food Chem., Kidlington, v. 89, p. 611-615, 2005.

[24] MORALES-AIZPURÚA, I.C.; TENUTA-FILHO, A. Óxidos de colesterol: ocorrência em alimentos, formação e efeitos biológicos. Rev. Bras. Ciênc. Farm., São Paulo, v. 38, n. 4, p. 431-442, 2002.

[25] NOVElli, E.; ZANARDI, E.; GHIRETTI, G.P.; CAMPANINI, G.; DAZZI, G.; MADARENA, G.; CHIZZOLINI, R. Lipid and cholesterol oxidation in frozen stored pork, salame milano and mortadella. Meat Sci., Oxford, v. 48, n.1/2, p. 29-40, 1998.

[26] PANIANGVAIT, P.; KING, A.J.; JONES, A.D.; GERMAN, B.G. Cholesterol oxides in foods of animal origin. J. Food Sci., Chicago, v. 60, n. 6, p. 1159-1174, 1995.

[27] RAZZAZI-FAZELI, E.; KLEINEISEN, S.; LUF, W. Determination of cholesterol oxides in processed food using high-performance liquid chromatography-mass spectrometry with atmospheric pressure chemical ionization. J. Chromatogr., A., Amsterdam, v. 896, p. 321-334, 2000.

[28] SÃO PAULO. Código Sanitário: Decreto no 12.486 de 28 de outubro de 1978, 5. Ed., p. 259-269, São Paulo, 1992.

[29] SARANTINOS, J.; O'DEA, K.; SINCLAIR, A.J. Cholesterol oxides in Australian foods: identification and quantification. Food Aust., North Sydney, v. 45, n.10, p. 485-490, 1993.

[30] SEVANiAn, A.; PETERSON, A.R. The cytotoxic and mutagenic properties of cholesterol oxidation products. Food Chem. Toxicol., Oxford, v. 24, n. 10/11, p. 11031110, 1986.

[31] SEVANIAN, A.; SERAGLIA, R.; TRALDI, P.; ROSSATO, P.; URSINI, F.; HODIS, H. Analysis of plasma cholesterol oxidation products using gas- and high-performance liquid chromatography/mass spectrometry. Free Rad. Biol. \& Med., New York, v. 17, n. 5, p. 397-409, 1994.

[32] SMITH, L.L. Review of progress in sterol oxidation: 19871995. Lipids, Champaign, v. 31, n. 5, p. 453-487, 1996.

[33] STAUFFER, C.E. Fats and oils. Saint Paul: Eagan Press. $149 \mathrm{p}, 1996$.

[34] TAI, C-Y.; CHEN, YC.; CHEN, B.H. Analysis, formation and inhibition of cholesterol oxidation products in food: An overview (Part I). J. Food Drug Anal., Nankang, v. 7, n. 4, p. 243-257,1999.

[35] TAI, C-Y.; CHEN, YC.; CHEN, B.H. Analysis, formation and inhibition of cholesterol oxidation products in food: An overview (Part II). J. Food Drug Anal., Nankang, v.8, n. 1, p. 1-15, 2000.

[36] TENUTA-FILHO, A.; MORALES-AIZPURÚA, I.C.; MOURA, A.F.P.; KITAHARA, S.E. Óxidos de colesterol em alimentos. Rev. Bras. Ciênc. Farm., São Paulo, v. 39, n. 3, p. 319-325, 2003.

\section{6 - AGRADECIMENTOS}

À FAPESP e ao CNPq, pelo apoio recebido na execução do projeto de pesquisa. 